\begin{tabular}{lrr} 
STUDIA & ROMANICA & POSNANIENSIA \\
\hline UAM & Vol. $41 / 1$ & Poznań 2014
\end{tabular}

MARJANA ŠIFRAR KALAN

Universidad de Ljubljana

\title{
DISPONIBILIDAD LÉXICA EN DIFERENTES NIVELES DE ESPAÑOL/LENGUA EXTRANJERA
}

\begin{abstract}
A bstract. Marjana Šifrar Kalan, Disponibilidad léxica en diferentes niveles de español/lengua extranjera. [Lexical Availability in different levels of Spanish as a Foreign Language], Studia Romanica Posnaniensia, Adam Mickiewicz University Press, Poznań, vol. XLI/1: 2014, pp. 63-85. ISBN 978-83-232-2673-4. ISSN 0137-2475. eISSN 2084-4158. Doi: 10.7169/strop2014.411.005

The objective of this study is to present the lexical availability in Slovene students of Spanish FL through the application of lexico-statistical methodology. The lexical availability of eleven semantic categories is compared quantitatively and qualitatively in two different level groups of students of Spanish FL: 100 high-school students (with approximate level B1) and 100 university students (with approximate level B2+). The similarities and differences according to different levels of Spanish are presented.
\end{abstract}

Keywords: Spanish/FL, lexical availability, high-school Spanish students, university Spanish students, semantic catagories

\section{INTRODUCCIÓN}

Los estudios de disponibilidad léxica nacieron a mediados del siglo XX en Francia como complemento de los léxicos básicos o de frecuencia con el fin de seleccionar los vocablos que deben ser enseñados en francés como lengua extranjera. La disponibilidad léxica ha abierto las puertas a la realización de diferentes estudios. En español, el método fue adoptado y adaptado por Humberto López Morales, el coordinador del gran proyecto panhispánico que tiene como objetivo elaborar diccionarios de disponibilidad léxica para diversas zonas del mundo hispánico (Bartol y Hernández, 2003 www.dispolex.com), ya que los trabajos de disponibilidad léxica tienen como objetivo principal conocer el léxico disponible de un grupo determinado de hablantes. El léxico disponible está compuesto por aquellas palabras que sólo aparecen en situaciones precisas, y cuando el tema de conversación las requiere: razón por la que también se las denomina «palabras temáticas». De este modo queda claro que las palabras disponibles son palabras muy conocidas, pero no muy frecuentes. El léxico disponible, junto con el léxico básico, que abarca las palabras más frecuentes y estables de una comunidad, constituye el léxico fundamental según López Morales (1999: 20). 
En el aspecto metodológico el proyecto panhispánico se aleja de las pautas de las primeras investigaciones francesas y canadienses, que emplearon listas cerradas, y utiliza el sistema de listas abiertas de pruebas asociativas a partir de ciertos campos semánticos (también llamados centros de interés) que funcionan como estímulos verbales. A partir de estos estímulos los informantes escriben las palabras más disponibles que acuden de manera más rápida y fácil a la mente. Las aplicaciones de los métodos léxico-estadísticos han dado lugar a muchísimos estudios en el ámbito hispanohablante, tanto en España como en América Latina, pero a partir del año 2000 algunos hispanistas han aplicado la metodología de estos trabajos a informantes extranjeros de ELE fuera de España: Carcedo González (2000) en Finlandia, López González (2010) en Polonia, Jing (2012) en China, Magnúsdóttir (2012) en Islandia y González Fernández (2013) en Turquía. También en España se han hecho varias investigaciones a estudiantes extranjeros de ELE (Samper Hernández, 2002; Sánchez Gómez, 2005; López Rivero, 2008; Pérez Serrano, 2009; Fernández-Merino Gutiérrez, 2011; Sánchez-Saus Laserna, 2011; Jiménez Berrio, 2013).

Con nuestro estudio de disponibilidad léxica en estudiantes eslovenos de ELE queremos seguir esta línea de investigaciones aplicada a la enseñanza y al aprendizaje del léxico español. Los objetivos del presente trabajo son:

- conocer la disponibilidad léxica de los estudiantes eslovenos de ELE mediante la aplicación de la metodología léxico-estadística;

- comparar cuantitativamente la disponibilidad léxica en once campos semánticos de dos grupos de niveles diferentes de ELE: 100 estudiantes de secundaria (nivel aproximado B1) y 100 estudiantes universitarios (nivel aproximado B2+); y detectar: 1) la producción de todas las asociaciones; 2) la diversidad léxica o el número de vocablos (palabras diferentes); 3) el promedio de palabras por informante; 4) la cohesión de los campos semánticos; 5) el número de palabras más disponibles; 6) las palabras comunes; $y$

- comparar cualitativamente la disponibilidad léxica de los dos grupos de estudiantes de ELE en once campos semánticos y detectar las diferencias y similitudes léxicas según el conocimiento de ELE.

Nos proponemos comprobar la validez de las siguientes hipótesis:

1) La disponibilidad léxica de los estudiantes de secundaria con menos conocimiento de español es inferior a la de los estudiantes universitarios.

2) En ambos grupos, los mismos campos semánticos provocan un mayor número de asociaciones.

3) Mayoritariamente, las asociaciones corresponden a la misma clase gramatical.

4) Los estudiantes con más conocimiento (estudiantes universitarios) producen asociaciones más estrechamente conectadas con el tema que los estudiantes con menos conocimiento de español (estudiantes de secundaria).

5) Existe similitud asociativa fuerte en los prototipos semánticos (las palabras más disponibles) de los dos grupos. 


\section{DATOS BÁSICOS Y METODOLOGÍA}

Siguiendo el procedimiento tipificado y estudiado por la psicolingüística como asociación controlada, en el que se basan los estudios de disponibilidad léxica, se ha recogido el vocabulario de once campos semánticos. Como el objetivo es observar la evolución léxica desde el nivel secundario hasta el grado superior de especialización universitaria, análogamente al estudio realizado por Carcedo González (2000) entre los estudiantes finlandeses, la muestra está compuesta por dos grupos de informantes: 1) 100 alumnos (de 17 o 18 años) del final del tercer curso ${ }^{1}$ de cuatro institutos eslovenos de Educación Secundaria escogidos entre casi $4000^{2}$ alumnos que en los años de recogida de datos estudiaban español y tenían un nivel aproximado de B1;2) 100 estudiantes de Filología Hispánica de la Facultad de Letras de la Universidad de Liubliana (de 20 a 25 años), quienes tenían un nivel aproximado de B2+ ${ }^{3}$. La encuesta se llevó a cabo en las clases de español en los institutos (IES) y en la universidad durante la primavera de 2006 y 2007. Se han seguido las pautas metodológicas comunes del proyecto panhispánico del léxico disponible (López Morales, 1999), pero con algunos cambios que reflejan las características de la muestra de ELE y no de la lengua materna, como es el caso del proyecto panhispánico. Por ello se han descartado las variables de índole sociológica ${ }^{4}$ destacándose sobre todo el nivel de conocimiento de ELE, en tanto que tal es el objeto de estudio. Los campos semánticos contemplados en esta investigación (Šifrar Kalan, 2011) son los siguientes: 1) Partes del cuerpo, 2) La ropa, 3) La casa, 4) Alimentos y bebidas, 5) La ciudad, 6) El campo, 7) Medios de transporte, 8) Los animales, 9) Juegos y distracciones, 10) Profesiones y oficios, 11) Acciones que se realizan todos los días. De los 16 centros de interés tradicionalmente investigados en disponibilidad léxica se han descartado aquellos campos semánticos que se han mostrado como menos productivos tanto en las investigaciones de disponibilidad léxica en ELE (Carcedo González, 2000: 93; Samper Hernández, 2002: 48; López González, 2010: 5), como también en español como lengua materna (López Morales, 1999: 41; Carcedo González, 2001: 58; González Martínez, 2002: 22; Samper Padilla, Bellón Fernández, Samper Hernández, 2003: 59; Prado Aragonés y Galloso Camacho, 2005: 56), y que ya no reflejan fielmente el léxico de la sociedad del siglo XXI, ni los intereses de los jóvenes, quienes son el objeto de estudio en las investigaciones de

${ }^{1}$ La muestra se escogió entre los alumnos de ELE con aproximadamente 300 horas de español, que tenían la asignatura de español en el ámbito escolar.

${ }^{2}$ El número de casi 4000 alumnos se refiere a todos los alumnos que en los IES estudiaron español como segunda o tercera lengua extranjera desde el primero hasta el cuarto año de secundaria.

${ }^{3}$ La mayoría de los estudiantes universitarios había tenido por lo menos 600 horas de español. En los dos grupos se trata de aprendizaje escolar y no de inmersión.

${ }^{4}$ Se ha descartado la variable del sexo porque la gran mayoría de los informantes son chicas y la variable del centro privado o público porque en Eslovenia prevalece la educación pública. En realidad, Carcedo González (2000) y Samper Hernández (2002) informan de que el sexo no ejerce ninguna influencia importante. 
disponibilidad léxica. Se trata de campos semánticos que, en la mayoría de los casos, no se presentan en los manuales de ELE. Samper Padilla, Bellón Fernández y Samper Hernández (2003), quienes comparan los resultados de distintas investigaciones del proyecto panhispánico, también observan que los campos asociativos que nunca consiguen sobrepasar la media de respuestas (Partes de la casa, Objetos colocados en la mesa para la comida, Los muebles de la casa, Iluminación y calefacción, Trabajos del campo y del jardín) presentan «áreas léxicas más cerradas y poco familiares para los informantes de este tipo de estudio» (2003: 59). Hemos añadido un nuevo centro de interés, Acciones que se realizan todos los días, ya propuesto por los investigadores de la escuela canadiense Mackey y Azurmendi (Samper Padilla, Bellón Fernández, Samper Hernández, 2003: 56). Se ha optado por este centro para poder observar el léxico más frecuente en los manuales de ELE y comprobar si las asociaciones corresponden a la clase gramatical del estímulo (en este caso, los verbos, dado que otros campos semánticos son indicados por los sustantivos).

Para recoger las asociaciones se usó el mismo tiempo límite que en otras investigaciones: dos minutos para cada campo semántico. Para unificar, eliminar y corregir el léxico recogido hemos seguido los criterios de los investigadores de disponibilidad léxica en español como lengua materna (Samper Padilla, 1998; Carcedo González, 2001; Gómez Molina y Gómez Devís, 2004) y en ELE (Carcedo González, 2000; Samper Hernández, 2002), y para informatizarlo, nos hemos servido del programa DISPOLEX (Bartol y Hernández, 2003 www.dispolex.com).

\section{ANÁLISIS CUANTITATIVO Y COMPARATIVO DE LOS INFORMANTES ESLOVENOS}

Las tablas 1 y 2 presentan los resultados generales del léxico disponible de dos grupos de informantes eslovenos con diferentes niveles de conocimiento de ELE: los estudiantes de secundaria y los estudiantes universitarios.

Tabla 1. Resultados del léxico disponible de estudiantes de secundaria

\begin{tabular}{|l|c|c|c|c|}
\hline \multicolumn{1}{|c|}{ Centro de interés } & $\begin{array}{c}\text { Palabras } \\
\text { (rango) }\end{array}$ & $\begin{array}{c}\text { Vocablos } \\
\text { (rango) }\end{array}$ & $\begin{array}{c}\text { Promedio/ } \\
\text { Informante }\end{array}$ & $\begin{array}{c}\text { Índice } \\
\text { de cohesión }\end{array}$ \\
\hline 1. Partes del cuerpo & $1209(7)$ & $128(11)$ & 12,09 & 0,13 \\
\hline 2. La ropa & $986(11)$ & $164(10)$ & 9,86 & 0,09 \\
\hline 3. La casa & $1299(5)$ & $290(5)$ & 12,99 & 0,06 \\
\hline 4. Alimentos y bebidas & $1544(2)$ & $274(6)$ & 15,44 & 0,04 \\
\hline 5. La ciudad & $1434(3)$ & $330(1)$ & 14,34 & 0,04 \\
\hline 6. El campo & $1122(9)$ & $269(7)$ & 11,22 & 0,04 \\
\hline
\end{tabular}


tabla 1 continua

\begin{tabular}{|l|c|c|c|c|}
\hline 7. Medios de transporte & $1077(10)$ & $168(9)$ & 10,77 & 0,06 \\
\hline 8. Los animales & $1162(8)$ & $245(8)$ & 11,62 & 0,05 \\
\hline 9. Juegos y distracciones & $1393(4)$ & $291(4)$ & 13,93 & 0,05 \\
\hline 10. Profesiones y oficios & $1267(6)$ & $302(3)$ & 12,67 & 0,04 \\
\hline $\begin{array}{l}\text { 11. Acciones que se realizan } \\
\text { todos los días }\end{array}$ & $1602(1)$ & $309(2)$ & 16,02 & 0,05 \\
\hline Promedio total & 1281 & 252 & 12,8 & 0,053 \\
\hline
\end{tabular}

Tabla 2. Resultados del léxico disponible de estudiantes universitarios

\begin{tabular}{|c|c|c|c|c|}
\hline Centro de interés & $\begin{array}{l}\text { Palabras } \\
\text { (rango) }\end{array}$ & $\begin{array}{l}\text { Vocablos } \\
\text { (rango) }\end{array}$ & $\begin{array}{l}\text { Promedio/ } \\
\text { Informante }\end{array}$ & $\begin{array}{c}\text { Índice } \\
\text { de cohesión }\end{array}$ \\
\hline 1. Partes del cuerpo & $1907(2)$ & $145(11)$ & 19,07 & 0,13 \\
\hline 2. La ropa & $1196(11)$ & $193(10)$ & 11,96 & 0,06 \\
\hline 3. La casa & $1855(3)$ & $285(6)$ & 18,55 & 0,07 \\
\hline 4. Alimentos y bebidas & $2011(1)$ & $328(3)$ & 20,11 & 0,06 \\
\hline 5. La ciudad & $1644(5)$ & $343(1)$ & 16,44 & 0,05 \\
\hline 6. El campo & $1470(7)$ & $333(2)$ & 14,70 & 0,04 \\
\hline 7. Medios de transporte & $1223(10)$ & $197(9)$ & 12,23 & 0,06 \\
\hline 8. Los animales & $1519(6)$ & $213(8)$ & 15,19 & 0,07 \\
\hline 9. Juegos y distracciones & $1406(8)$ & $287(5)$ & 14,06 & 0,05 \\
\hline 10. Profesiones y oficios & $1396(9)$ & $263(7)$ & 13,96 & 0,05 \\
\hline $\begin{array}{l}\text { 11. Acciones que se realizan } \\
\text { todos los días }\end{array}$ & $1797(4)$ & $295(4)$ & 17,97 & 0,06 \\
\hline Promedio total & 1584 & 262 & 15,84 & 0,064 \\
\hline
\end{tabular}

\subsection{CANTIDAD TOTAL DE PALABRAS}

El grupo de estudiantes de secundaria ha producido menos palabras en total (1281 palabras en promedio por cada centro de interés) que los estudiantes universitarios, quienes han escrito unas 300 palabras más como promedio. En cuanto a los centros menos productivos, los dos grupos coinciden en La ropa y Medios de transporte. En los centros más productivos coinciden solo en Alimentos y bebidas, el centro con más palabras aportadas entre los estudiantes universitarios, y en el segundo lugar entre los estudiantes de secundaria, donde se han producido más palabras en Acciones que se realizan todos los días. En este caso, el mayor número de asociaciones puede que haya reflejado la enseñanza del español en secundaria, dado que en los niveles A1-B1 
se refuerzan mucho la comunicación y las actividades diarias ${ }^{5}$. El caso de los centros más productivos en el grupo con más conocimiento (Alimentos y bebidas, Partes del cuerpo, La casa) se puede relacionar con el manual Abanico ${ }^{6}$ usado por aquellos informantes en el primer curso universitario, ya que el manual trata precisamente estos campos semánticos. Los centros menos productivos (La ropa, Medios de transporte) probablemente reflejan un carácter cerrado semánticamente. Como puede apreciarse en el gráfico 1, los estudiantes universitarios han escrito más palabras en cada uno de los centros.

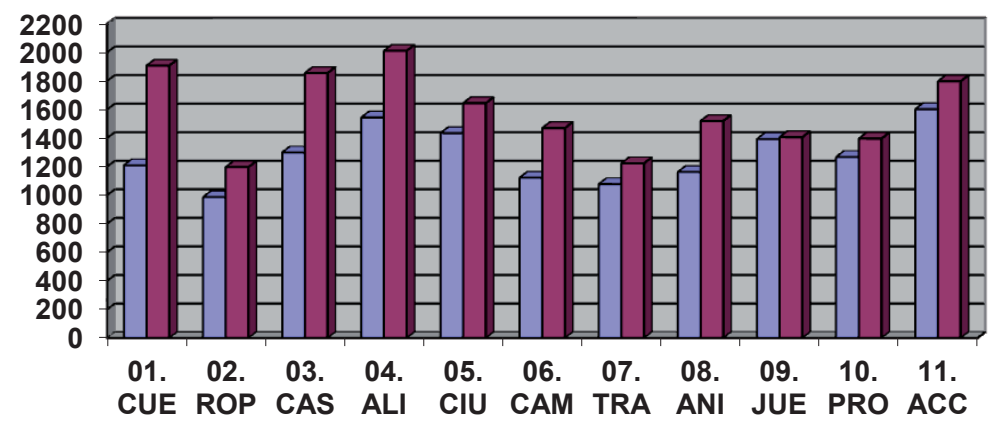

Gráfico 1. Comparación de todas las palabras producidas entre los estudiantes de secundaria (SEC) y los estudiantes universitarios (UNI) en once centros de interés

Las tablas 1 y 2 también reflejan el promedio de palabras por informante, que es un dato muy fiable de la productividad léxica porque es comparable con otras investigaciones a pesar de la diferencia de número de informantes. Los promedios en los estudiantes de secundaria oscilan entre 9,86 en La ropa y 16 palabras por informante en el centro Acciones que se realizan todos los días, seguido inmediatamente por el centro Alimentos y bebidas con 15,44 palabras de promedio. Los informantes universitarios han producido una media de 20,11 palabras en Alimentos y bebidas, 19,07 en Partes del cuerpo, 18,55 en La casa, pero solo 11,96 en La ropa. Aunque la comparación con otras investigaciones no es el objetivo de este artículo ${ }^{7}$, cabe añadir que en los trabajos panhispánicos los resultados de los diez centros de interés comunes superan casi siempre el número de 20 unidades por informante, mientras que en los centros de interés que en este estudio se han descartado los promedios son más bajos (Samper Padilla, Bellón Fernández, Samper Hernández, 2003: 57).

${ }^{5}$ La mayoría de los estudiantes de instituto utilizaba los manuales Gente 1 y Gente 2, de la editorial Difusión.

${ }^{6}$ Abanico. Curso avanzado de español. Difusión.

7 Para más detalles sobre las comparaciones de este estudio con otros, véanse Šifrar Kalan (2009) y Šifrar Kalan (2012a). 


\subsection{PRODUCTIVIDAD DE VOCABLOS O DIVERSIDAD LÉXICA}

Tanto las tablas 1 y 2 como el gráfico 2 muestran que en los dos grupos observados no hay tanta diferencia en la diversidad léxica (el número de palabras diferentes) como en la producción léxica. Los estudiantes de instituto han escrito 252 palabras diferentes, de media y por centro de interés, y los universitarios 262. La diversidad léxica según los centros de interés no difiere mucho en los dos grupos a pesar de los niveles diferentes de conocimiento de español, porque parece que está más relacionada con el carácter difuso o cerrado del centro de interés. Se ha confirmado que Partes del cuerpo, La ropa, Medios de transporte son centros compactos o cerrados, y La ciudad el centro más abierto o difuso que provoca asociaciones muy diferentes. El gráfico 2 muestra que en cinco centros de interés el número de vocablos obtenidos por los estudiantes de secundaria es algo superior a aquellos producidos por los universitarios, lo que se debe a las asociaciones secundarias, más dispersas entre los estudiantes con menos nivel. Las diferencias son más grandes en los centros Profesiones y oficios y Los animales. Por el contrario, los estudiantes universitarios destacan con una mayor diversidad léxica en El campo, Alimentos y bebidas, La ropa y Medios de transporte.

Estos resultados han confirmado las conclusiones de otras investigaciones de disponibilidad léxica en ELE (Carcedo González, 2000: 94; Samper Hernández, 2002: 49; López González, 2010: 6) y en español como lengua materna (López Morales, 1999: 41; Carcedo González, 2001: 59; Samper Padilla, Bellón Fernández, Samper Hernández, 2003: 62): «[...] ya puede deducirse que la mayor o menor riqueza de vocablos no se corresponde con la productividad de unidades léxicas en las diferentes áreas temáticas; es decir, que no se cumple la máxima: a mayor número de respuestas, mayor número de palabras diferentes» (Gómez Molina y Gómez Devís, 2004: 76-77).

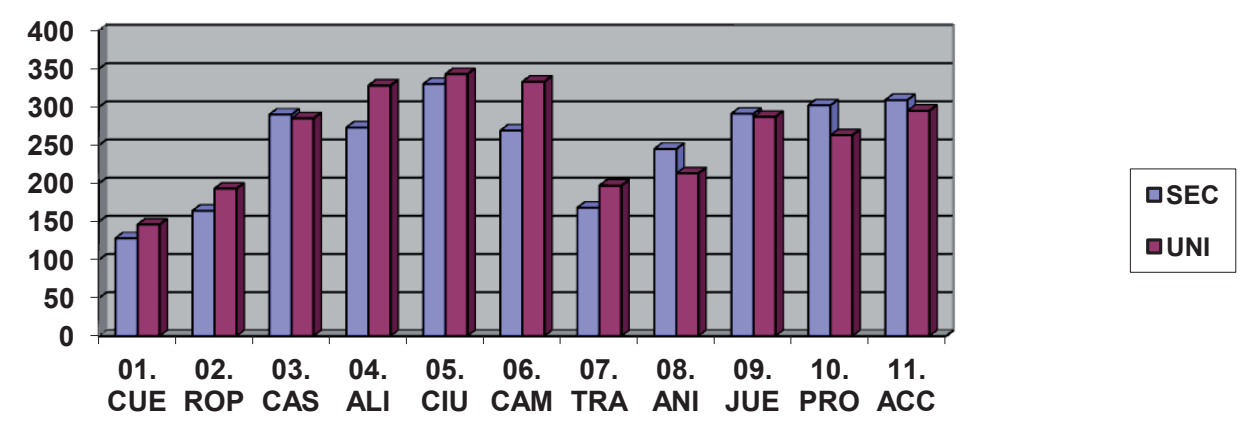

Gráfico 2. Comparación de palabras diferentes entre los estudiantes de secundaria (SEC) y los estudiantes universitarios (UNI) en once centros de interés 


\subsection{COHESIÓN DE LOS DATOS}

Con el índice de cohesión ${ }^{8}$ podemos conocer las tendencias de conjunto de la muestra, precisamente podemos observar qué centros de interés son más cerrados o compactos (el valor 1 significaría que todos los informantes han escrito las mismas palabras) y cuáles son más abiertos o dispersos (el valor 0 significaría que todos han escrito palabras diferentes). En el grupo de estudiantes de secundaria los índices oscilan entre 0,04 y 0,13 , el promedio de todos los centros es 0,053 ; en el grupo universitario los índices tienen completamente la misma oscilación, pero el promedio de 0,064 es un poco más alto, lo cual indica que los estudiantes universitarios han dado más respuestas iguales que los estudiantes de secundaria. Según la clasificación de cohesión de los centros de interés que proponen Gómez Molina y Gómez Devís (2004: 83), podemos clasificar los once centros de interés de este estudio de la manera siguiente:

a) campos semánticamente más compactos (mayor concreción semántica; índice $\geq 0,06)$ en el grupo de estudiantes de secundaria: Partes del cuerpo, La ropa, La casa, Medios de transporte; en los estudiantes universitarios: Partes del cuerpo, La ropa, La casa, Alimentos y bebidas, Medios de transporte, Los animales, Acciones que se realizan todos los días.

b) centros de interés con un grado medio de coincidencia en las respuestas (índice $\sim 0,05)$ en el grupo de estudiantes de secundaria: Los animales, Juegos y distracciones, Acciones que se realizan todos los dias; en los estudiantes universitarios: La ciudad, Juegos y distracciones, Profesiones y oficios.

c) campos semánticamente más difusos (índice $\leq 0,04$ ) en el grupo de estudiantes de secundaria: Alimentos y bebidas, La ciudad, El campo, Profesiones y oficios; en los estudiantes universitarios: El campo.

En los dos grupos destaca como el campo semánticamente más compacto Partes del cuerpo con el mismo índice de 0,13 . Se trata de un área temáticamente muy uniformada, donde las asociaciones no dependen de factores sociales y culturales, mientras que los centros La ciudad y El campo, los centros más difusos, pueden provocar asociaciones muy diferentes dependiendo de los factores sociales, culturales y geográficos. Estos resultados coinciden con las investigaciones llevadas a cabo con hispanohablantes (Gómez Molina y Gómez Devís, 2004: 84-85; Samper Padilla, Bellón Fernández, Samper Hernández, 2003).

Cabe añadir que a los informantes eslovenos, al comienzo de la prueba, se les proporcionó, entre otras instrucciones, que escribieran todas las palabras que les vinieran a la mente sobre el tema propuesto, que trataran de seguir su flujo de pensamiento. Si hubieran recibido las instrucciones de escribir solo las palabras estrechamente conectadas con el tema, los resultados hubieran sido diferentes y los centros más compactos.

${ }^{8}$ Se calcula dividiendo el promedio de palabras por informante en cada centro de interés entre el número de palabras diferentes. 


\subsection{DISPONIBILIDAD DE PALABRAS}

Los datos presentados en los capítulos anteriores se basan en todas las palabras escritas por los 200 informantes, desde las más disponibles que han escrito muchos estudiantes hasta las menos disponibles que solo han escrito algunos informantes. Para evitar las comparaciones individuales y el «error» metodológico y resaltar los resultados colectivos, hay que contrastar solo las palabras más disponibles. Como resume Samper Hernández (2003: 969), algunos investigadores optaron por las primeras 50 palabras, otros por las palabras con un $75 \%$ de disponibilidad acumulada o según los índices de disponibilidad. En la tabla 3 se presenta el número de vocablos que superan el 0,1 y el 0,02 en el índice de disponibilidad, y los vocablos con frecuencia acumulada del $75 \%$ de la muestra. El número mayor de vocablos es el que tiene un índice mayor a 0,02 y oscila entre 37 vocablos (Partes del cuerpo, estudiantes de secundaria) y 108 (Alimentos y bebidas, estudiantes universitarios). Se puede observar que el centro de interés con más palabras disponibles en los estudiantes de secundaria es La ciudad, seguido por Juegos y distracciones y Alimentos y bebidas. En la gran mayoría de los centros de interés el número de vocablos más disponibles del grupo universitario supera al grupo de secundaria.

Tabla 3. Resultados según el índice de disponibilidad (SEC - estudiantes de secundaria; UNI - estudiantes universitarios)

\begin{tabular}{|c|c|c|c|c|c|c|}
\hline \multirow[t]{2}{*}{ Centro de interés } & \multicolumn{2}{|c|}{$\begin{array}{c}\mathrm{N}^{\circ} \text { de vocablos } \\
\text { con índice de } \\
\text { disponibilidad } \\
>0,1\end{array}$} & \multicolumn{2}{|c|}{$\begin{array}{l}\mathrm{N}^{\circ} \text { de vocablos } \\
\text { con índice de } \\
\text { disponibilidad } \\
>0,02\end{array}$} & \multicolumn{2}{|c|}{$\begin{array}{c}\mathrm{N}^{\circ} \text { de vocablos } \\
\text { con frecuencia } \\
\text { acumulada del } \\
75 \%\end{array}$} \\
\hline & SEC & UNI & SEC & UNI & SEC & UNI \\
\hline 1. Partes del cuerpo & 21 & 28 & 37 & 51 & 18 & 26 \\
\hline 2. La ropa & 14 & 16 & 51 & 55 & 34 & 33 \\
\hline 3. La casa & 20 & 25 & 80 & 92 & 69 & 66 \\
\hline 4. Alimentos y bebidas & 24 & 23 & 84 & 108 & 62 & 79 \\
\hline 5. La ciudad & 16 & 18 & 87 & 90 & 89 & 80 \\
\hline 6. El campo & 16 & 22 & 68 & 87 & 64 & 78 \\
\hline 7. Medios de transporte & 12 & 10 & 50 & 50 & 32 & 35 \\
\hline 8. Los animales & 14 & 22 & 60 & 60 & 51 & 35 \\
\hline 9. Juegos y distracciones & 17 & 20 & 85 & 85 & 70 & 70 \\
\hline 10. Profesiones y oficios & 20 & 23 & 74 & 74 & 75 & 57 \\
\hline $\begin{array}{l}\text { 11. Acciones que se realizan } \\
\text { todos los días }\end{array}$ & 20 & 29 & 81 & 82 & 69 & 57 \\
\hline
\end{tabular}


Para el análisis cuantitativo de vocablos comunes entre los dos grupos hemos optado por el límite del índice 0,02. Con esta selección de palabras disponibles (tabla 4) resulta que los dos grupos de informantes tienen mayor número de vocablos comunes en Juegos y distracciones (58,88\%), Medios de transporte (58,73\%) y Partes del cuerpo $(57,14 \%)$. El menor grado de convergencia se encuentra en los centros La casa y La ropa.

Tabla 4. Vocablos comunes con un índice de disponibilidad mayor a 0,02 en los dos grupos observados

\begin{tabular}{|l|c|c|c|}
\hline \multicolumn{1}{|c|}{ Centro de interés } & $\begin{array}{c}\mathrm{N}^{\circ} \text { de vocablos } \\
\text { con I.D. }>0,02, \\
\text { estudiantes de SEC }\end{array}$ & $\begin{array}{c}\mathrm{N}^{\circ} \text { de vocablos } \\
\text { con I.D. }>0,02, \\
\text { estudiantes de UNI }\end{array}$ & $\begin{array}{c}\text { Vocablos comunes } \\
{[\%]}\end{array}$ \\
\hline Juegos y distracciones & 85 & 85 & 58,88 \\
\hline Medios de transporte & 50 & 50 & 58,73 \\
\hline Partes del cuerpo & 37 & 51 & 57,14 \\
\hline $\begin{array}{l}\text { Acciones que se realizan } \\
\text { todos los días }\end{array}$ & 81 & 82 & 53,77 \\
\hline El campo & 68 & 87 & 51,96 \\
\hline Profesiones y oficios & 74 & 74 & 51,02 \\
\hline Alimentos y bebidas & 84 & 60 & 48,84 \\
\hline Los animales & 80 & 90 & 46,34 \\
\hline La ciudad & 80 & 92 & 46,28 \\
\hline La casa & 51 & 55 & 45,76 \\
\hline La ropa & & & 45,21 \\
\hline
\end{tabular}

\section{ANÁLISIS CUALITATIVO}

A partir de los datos presentados se ha hecho un análisis cualitativo con dos grupos de vocablos: 1) todos los vocablos cuyo índice supera el 0,02, y 2) los primeros 20 vocablos de mayor disponibilidad de cada centro de interés (Šifrar Kalan, 2011). Por falta de espacio en este artículo, se presentan las tablas del segundo análisis (véase Anexo). Se trata de las primeras y las más frecuentes asociaciones producidas por los estudiantes eslovenos con dos niveles diferentes de conocimiento de español. El índice de disponibilidad indica cuántos estudiantes han escrito la palabra y en qué posición. Las palabras en negrita son aquellas que en el otro grupo no figuran entre las primeras 20 pero pueden figurar a partir de ese puesto.

Partes del cuerpo es el centro con mayor convergencia entre los dos grupos. Entre las primeras 20 coinciden 17 palabras, y en la lista con el índice mayor a 0,02 
coinciden el $57 \%$ de las palabras. Se trata solo de sustantivos, a excepción del verbo doler en los estudiantes de secundaria. Las palabras que están en la lista (I.D. > 0,02) de los universitarios y no en la lista de los estudiantes de secundaria son aquellas que pertenecen a los niveles más altos de conocimiento de la lengua extranjera, como, por ejemplo: ceja, ombligo, párpado, cabello, muslo, nalga, pómulo...

En el centro La ropa hay menos palabras comunes: 12 entre las primeras 20 y el $45 \%$ en la lista I.D. $>0,02$. Las diferencias se deben fundamentalmente a la presencia de adjetivos, sobre todo relativos a colores en la lista de los estudiantes de secundaria, mientras que en la lista de los estudiantes universitarios solo hay dos colores hasta el índice 0,02. Para los estudiantes con menos conocimiento de español los colores pueden constituir un vocabulario más básico y frecuente que algunas prendas de vestir, por eso escribieron los colores como primeras asociaciones. Algunas palabras (I.D. $>0,02)$ que no se encuentran en la lista de los estudiantes de secundaria son media, boina, planchar.

La convergencia en La casa es también del $45 \%$ y 15 palabras entre las primeras 20. En este caso la diferencia se debe a muchas palabras que indican miembros de la familia en la lista de los estudiantes de secundaria. Se trata de nuevo del vocabulario más básico o consolidado que el de algunas partes de la casa o los muebles, que es lo que presenta el vocabulario más disponible entre los universitarios.

En las listas de los dos grupos en el centro Alimentos y bebidas ${ }^{9}$ encontramos verbos (comer, beber, cocinar, comprar, freir) y sintagmas nominales (jamón serrano, vino blanco, vino tinto, patata frita, zumo de naranja, pulpo a la gallega, bebida alcohólica). En los dos grupos, las palabras más disponibles son las relativas a bebidas (agua, vino, zumo, cerveza), entre las palabras más disponibles referidas a comidas encontramos hiperónimos (fruta, verdura, carne).

La ciudad, como centro abierto, presenta uno de los porcentajes más bajos de palabras comunes en la lista de I.D. > 0,02 (46\%), mientras que hay mucha uniformidad en las palabras más disponibles: de las primeras 10 palabras coinciden 9. Entre las palabras de los estudiantes de secundaria hay más palabras generales y básicas, y menos estrechamente conectadas con el tema (habitante, amigo), además hay muchos verbos: 14 en una lista de 87 palabras con I.D. > 0,02 (pasar, girar, pasear, caminar, ir por la calle, comprar, vivir...). Las asociaciones de los estudiantes universitarios son más específicas y están más estrechamente conectadas con el tema y con su estilo de vida (alcalde, ayuntamiento, facultad, universidad). Solo hay 4 verbos en una lista de 90 palabras con I.D. $>0,02$.

En el centro El campo hay un 52\% de convergencia. Destacan los siguientes puntos comunes en el léxico disponible de los dos grupos: a) muchos sintagmas nominales (gente amable, aire fresco, gente amable, zona verde...), b) personas asociadas con el campo (campesino, abuelo, gente amable, familia, vecino...), c) conceptos abstractos (tranquilidad, paz, silencio, amor). Esto confirma que los informantes eslovenos tienen un concepto muy parecido del campo. Las palabras con I.D. > 0,02 que solo

\footnotetext{
9 Para más comentarios sobre este campo semántico, véase Šifrar Kalan (2012b).
} 
han producido los universitarios pertenecen a niveles de lengua más altos (hierba, trigo, finca, planicie, arroyo).

Medios de transporte, siendo un centro compacto, muestra un alto grado de convergencia (58,73\%). Asimismo, en las primeras 10 palabras más disponibles, las dos listas coinciden en 9 palabras comunes. Es el único centro donde los estudiantes universitarios han producido más verbos $(20 \%$ de la lista I.D. $>0,02)$ que los estudiantes de secundaria ( $12 \%$ de la lista I.D. $>0,02)$.

A pesar de la no muy alta convergencia en la lista I.D. $>0,02$ del centro Los animales $(46 \%)$, las primeras cinco palabras de mayor disponibilidad son exactamente las mismas (perro, gato, vaca, caballo, pájaro). En la lista de los universitarios prevalecen nombres de animales, mientras que en la lista de los estudiantes de secundaria hay más asociaciones secundarias que no designan animales (amor, campo, amable, comida, comer, negro...).

Juegos y distracciones es el centro con mayor número de palabras comunes (casi 59\%). En las dos listas hay 85 palabras con I.D. > 0,02, así que 63 son comunes. Pero la convergencia no es tan destacada en las palabras más disponibles ( 6 palabras comunes entre las primeras 10). Como el tema se relaciona con actividades, aparecen muchos verbos y sintagmas verbales: $33 \%$ en los estudiantes de secundaria y $31 \%$ en los estudiantes universitarios. Los subtemas en los que se pueden dividir las respuestas son: deporte, música, fiesta, cultura.

Profesiones y oficios presenta una convergencia media (51\%) y bastante baja en las primeras 20 palabras (6 palabras comunes de 10 ; 12 de 20). Nuevamente, las asociaciones de los universitarios están más estrechamente conectadas con el tema que en los estudiantes de secundaria. Las respuestas de los universitarios reflejan la relación con las letras propia de su carrera (poeta, intérprete, filósofo, licenciado).

En el centro Acciones que se realizan todos los días, casi el 54\% de las palabras son comunes. En este caso, al contrario de lo ocurrido en los demás centros, los estudiantes universitarios han producido más verbos y sintagmas verbales $(76 \%)$ que los estudiantes de secundaria (68\%). A pesar de la presencia de muchas palabras comunes, estas pueden tener índices muy diferentes, dependiendo de sus intereses y actividades. Nos encontramos con mucha diversidad temática, variedad que refleja la diversificación de las actividades diarias. Así, los estudiantes de secundaria han escrito palabras que solo pocos universitarios han escrito (profesor, padre, hermano, gato), y al revés, las palabras que abundan en el caso de los universitarios son menos frecuentes entre los estudiantes de secundaria (clase, universidad, facultad).

\subsection{LOS PROTOTIPOS COMUNES}

En las tablas con las primeras 20 palabras (Anexo) se ve claramente que el grado de convergencia de los prototipos en los dos grupos es muy elevado. En Partes del cuerpo las palabras más disponibles son: ojo, cabeza, mano. Pantalón y camisa son definitivamente los prototipos de La ropa. Cocina es el prototipo de La casa. En la 
categoría semántica Alimentos y bebidas, los dos léxicos de mayor disponibilidad presentan solo bebidas y ninguna palabra referente a comida, agua es la palabra más disponible. En la categoría abierta La ciudad coinciden los prototipos coche y calle. Otra categoría semántica abierta es El campo, donde prevalece el prototipo animal y naturaleza. En Medios de transporte, una categoría más cerrada, el prototipo es coche; en el campo semántico Animales los prototipos universales son, sin duda, perro y gato y también un animal muy común en Eslovenia, vaca. En Juegos y distracciones no prevalece ningún prototipo, hay dos palabras muy disponibles en los dos grupos: música y fútbol. Muchísima unanimidad hay también en el campo Profesiones y oficios, con los prototipos de profesor y médico. En el último centro de interés destacan como prototipos los verbos comer, dormir y estudiar.

Los lingüistas e investigadores del léxico disponible en ELE confirman que los informantes de diferentes niveles muestran gran convergencia en las palabras más disponibles, es decir, que a pesar de diferentes niveles de conocimiento de español, producen los mismos prototipos, lo que también se ha confirmado en el presente cotejo. Así, Jing (2012: 12) afirma que en los estudiantes chinos de español «también se observa un elevado grado de convergencia que se pone de manifiesto en los vocablos de la máxima disponibilidad léxica para los dieciséis centros de interés en los tres niveles de español sometidos a la prueba». Igualmente, sobre el cotejo de cuatro grupos de informantes (escolares y universitarios), Carcedo González (2000: 132) señala que «la convergencia del vocabulario entre los grupos es especialmente visible en los primeros puestos de la lista» y «que todo ello nos habla, de una gran similitud en el tipo de asociaciones que en los distintos estadios del aprendizaje establecen los sujetos finlandeses que componen la muestra» (2000: 134). Asimismo, López González (2010: 10) informa de una gran uniformidad en las unidades léxicas más disponibles en los dos grupos de niveles educativos diferentes.

\section{CONCLUSIONES}

El análisis del léxico disponible de los estudiantes eslovenos pone de relieve el grado de convergencia entre los dos grupos observados. Los resultados cuantitativos del léxico disponible de los estudiantes de secundaria (nivel aproximado B1) y de los estudiantes universitarios (nivel aproximado $\mathrm{B} 2+$ ) demuestran que la producción y la diversidad léxica dependen del nivel de conocimiento de una lengua extranjera. Tanto en el número de palabras escritas en total como en el número de palabras diferentes (vocablos) superan los estudiantes que tienen el nivel de español más alto, así que se confirma la primera hipótesis. La diferencia cuantitativa entre los dos grupos es más destacada en la producción de todas las palabras que en la diversidad léxica.

La comparación ha confirmado la similitud en la productividad solo en algunos grupos semánticos o centros de interés. En muchos estudios de disponibilidad léxica, igual que en este, los informantes han mostrado una de las mayores productividades de palabras en el campo sobre la comida y la bebida. En cuanto a los centros 
menos productivos, destacan en los dos grupos de informantes los centros La ropa y Medios de transporte.

Asimismo, se confirma la tercera hipótesis sobre las asociaciones de la misma clase gramatical que la presentada en el estímulo. Lo corrobora el predominio de los sustantivos en los estudios de disponibilidad léxica, ya que los nombres que designan los centros de interés son sustantivos. El mayor número de verbos y sintagmas verbales en los campos semánticos referentes a actividades o movimientos (Acciones que se realizan todos los días, Juegos y distracciones, Medios de transporte) señala adicionalmente que las asociaciones dependen del estímulo.

Considerando las listas completas del léxico disponible, los estudiantes universitarios han producido asociaciones que más fielmente corresponden al estímulo verbal, $\mathrm{y}$, consecuentemente, han escrito menos adjetivos, verbos y sintagmas nominales que los alumnos de secundaria. Estos últimos han producido menos asociaciones primarias y más asociaciones de otra categoría gramatical, probablemente debido a una riqueza léxica restringida. Comparando también la disponibilidad léxica de los extranjeros y los nativos (Šifrar Kalan, 2012a: 19) podemos concluir que habrá más asociaciones primarias cuanto más alto sea el nivel. Samper Hernández (2003: 980) también afirma que los estudiantes de ELE no producen tantas asociaciones primarias como los hispanohablantes. No cabe duda de que destacan las diferencias cualitativas, muy relevantes, en las respuestas de los dos grupos de informantes. La distinta «calidad» de las mismas constituye un inicio de madurez en el conocimiento de la lengua y la cultura hispánicas que no se ha alcanzado en los niveles de la enseñanza secundaria, en los que es frecuente encontrar aportaciones léxicas que solo indirectamente pueden asociarse con los correspondientes centros de interés.

Cualitativamente, además, se puede estimar que el léxico de mayor disponibilidad de los estudiantes con menor nivel de español y estudiantes con más nivel está en torno a la mitad; sin embargo, se observa muchísima uniformidad en las palabras con máxima disponibilidad, lo que confirma el carácter universal de los prototipos.

Por último, se pueden relacionar nuestras conclusiones con la organización del lexicón mental. Estamos de acuerdo con Schmitt (2000: 42) en que los estudiantes con mayor nivel de conocimiento y más asociaciones tienen un lexicón más organizado que los estudiantes con menor nivel.

\section{BIBLIOGRFÍA}

Bartol Hernández, José Antonio; Hernández Muñoz, Natividad (2003): Dispolex: Base de datos de la disponibilidad léxica. Salamanca: Universidad de Salamanca. Disponible en: http://www. dispolex.com [28.08.2013].

CARCedo González, Alberto (2000): Disponibilidad léxica en español como lengua extranjera: el caso finlandés (estudio del nivel preuniversitario y cotejo con tres fases de adquisición). Turku: Universidad de Turku.

Carcedo GonzÁlez, Alberto (2001): Léxico disponible de Asturias. Turku: Universidad de Turku. 
Fernández-Merino GutiérRez, Pablo Víctor (2011): «Presencia del léxico disponible de inmigrantes en glosarios específicos de vocabulario». Cuadernos Comillas, 2: 1-18.

Gómez Molina, José Ramón; Gómez Devís, Ma Begoña (2004): La disponibilidad léxica de los estudiantes preuniversitarios valencianos. Estudio de estratificación sociolingüística. Valencia: Universidad de Valencia.

GonZÁlez Fernández, Javier (2013): «La disponibilidad léxica de los estudiantes turcos de español como lengua extranjera». MarcoELE, 16. Disponible en: http://marcoele.com/disponibilidadlexica-de-estudiantes-turcos/ [28.08.2013].

GonzÁlez Martínez, Adolfo (2002): La disponibilidad léxica de los alumnos preuniversitarios de la provincia de Cádiz. Cádiz: Universidad de Cádiz.

JiMÉNEZ BERRIO, Felipe (2013): Léxico disponible de inmigrantes escolares no hispanohablantes. Pamplona: Servicio de Publicaciones de la Universidad de Navarra.

JING, Lin (2012): «El estudio de disponibilidad léxica de los estudiantes chinos de español como lengua extranjera». MarcoELE 14. Disponible en: http://marcoele.com/disponibilidad-lexicade-estudiantes-chinos/ [28 de agosto de 2013].

LÓPEZ GonZÁlez, Antonio María (2010): «La evaluación del desarrollo de la competencia léxica en L2 por medio de la disponibilidad léxica». Redele 18. Disponible en: http://www.mecd.gob.es/ redele/revistaRedEle/2010/primera.html [28.08.2013].

López Morales, Humberto (1999): Léxico disponible de Puerto Rico. Madrid: Arco Libros.

López Rivero, Eva (2008): Estudio de disponibilidad léxica en 43 estudiantes de ELE. Madrid: Universidad Antonio de Nebrija. Memoria de máster inédita.

MAGNúsDótTIR, Sigrún (2012): Disponibilidad léxica en alumnos de español como lengua extranje$\mathrm{ra}$. Estudio sobre el léxico disponible en alumnos de ELE en la secundaria en Islandia. Háskóli íslands: Sigillum Universitatis Islandiae. Memoria de máster inédita.

Pérez Serrano, Mercedes (2009): Estudio de disponibilidad léxica en estudiantes de E/LE en los centros de interés medios de transporte y profesiones y oficios. Instituto Cervantes y la Universidad Internacional Menéndez Pelayo. Memoria de Máster inédita.

Prado Aragonés, Josefina; Galloso Camacho, Ma Victoria (2005): Léxico disponible de Huelva: nivel preuniversitario. Huelva: Universidad de Huelva.

Samper Hernández, Marta (2002): Disponibilidad léxica en alumnos de español como lengua extranjera. Málaga: ASELE, Colección Monografías 4.

Samper Hernández, Marta (2003): «Datos comparativos entre el léxico disponible de estudiantes de español como lengua extranjera y el de la variedad grancanaria». In: Carmen Díaz Alayón, Marcial Morera, Gonzalo Ortega, [eds.], Estudios sobre el español de Canarias. Islas Canarias: Academia Canaria de la lengua, 961-987.

Samper Padilla, José Antonio (1998): «Criterios de edición del léxico disponible: sugerencias». Lingüistica, 10: 311-333.

Samper Padilla, José Antonio; Bellón Fernández, Juan José; Samper Hernández, Marta (2003): «El proyecto de estudio de la disponibilidad léxica en español». In: Gerd WotjaK [coord.], Pautas y pistas en el estudio del léxico hispano(americano). Madrid/Frankfurt: Iberoamericana/Vervuert, 27-140.

SÁnchez Gómez, Coronada (2005): «Naturaleza gramatical del léxico disponible en informantes de español como lengua extranjera». Interlingüística, 16/2: 977-986.

Sánchez-Saus LaSerna, Marta (2011): Bases semánticas para el estudio de los centros de interés del léxico disponible. Disponibilidad léxica de informantes extranjeros en las universidades andaluzas. Universidad de Cádiz. Tesis doctoral inédita.

Scнмiтt, Norbert (2000): Vocabulary in Language Teaching. Cambridge: Cambridge University Press. 
ŠIfrAR Kalan, Marjana (2009): «Disponibilidad léxica en español como lengua extranjera: el cotejo de las investigaciones en Eslovenia, Salamanca y Finlandia». Verba Hispánica, 17: 165-182.

ŠIFrar Kalan, Marjana (2011): Leksikalna razpoložljivost v španščini kot tujem jeziku. Ljubljana: Univerza $v$ Ljubljani. Tesis doctoral inédita.

ŠIFrar Kalan, Marjana (2012a): «Análisis comparativo de la disponibilidad léxica en español como lengua extranjera (ELE) y lengua materna (ELM) ». MarcoELE 15. Disponible en: http://marcoele.com/numeros/numero-15/ [28.08.2013].

ŠIfrar Kalan, Marjana (2012b): «El léxico disponible de comida y bebida». In: Anđelka Pejović, Mirjana Sekulić, Vladimir Karanović [eds.], Comida y bebida en la lengua española, cultura y literaturas hispánicas. Kragujevac: Facultad de Filología y Artes, 193-209.

\section{ANEXO}

Tablas de las primeras 20 palabras más disponibles en once centros de intéres (estudiantes de secundaria y estudiantes universitarios)

\begin{tabular}{|c|c|}
\hline \multicolumn{2}{|c|}{ 1. PARTES DEL CUERPO } \\
\hline Estudiantes sec & Estudiantes uni \\
\hline cabeza 0,76048 & cabeza 0,75838 \\
\hline mano 0,74563 & ojo 0,64828 \\
\hline ojo 0,64375 & mano 0,57581 \\
\hline pierna 0,46796 & pierna 0,54680 \\
\hline dedo 0,42116 & nariz 0,53357 \\
\hline nariz 0,40112 & dedo 0,46390 \\
\hline pelo 0,35398 & brazo 0,41914 \\
\hline rodilla 0,34148 & oreja 0,37082 \\
\hline espalda 0,31862 & labio 0,33230 \\
\hline boca 0,29072 & rodilla 0,29490 \\
\hline labio 0,27742 & pelo 0,29460 \\
\hline estómago 0,26564 & boca 0,29124 \\
\hline pie 0,23831 & espalda 0,27564 \\
\hline oreja 0,22198 & pie 0,27473 \\
\hline brazo 0,17055 & uña 0,26757 \\
\hline cuello 0,13675 & diente 0,24582 \\
\hline piel 0,11648 & cuello 0,23305 \\
\hline diente 0,11616 & hombro 0,21229 \\
\hline lengua 0,11261 & estómago 0,18979 \\
\hline pecho 0,10469 & muñeca 0,17294 \\
\hline
\end{tabular}




\begin{tabular}{|c|c|}
\hline Estudiantes sec & Estudiantes uni \\
\hline pantalón 0,69801 & pantalón 0,75474 \\
\hline camisa 0,62973 & camisa 0,69538 \\
\hline zapato 0,42259 & falda 0,55920 \\
\hline camiseta 0,36713 & camiseta 0,47215 \\
\hline falda 0,32568 & zapato 0,38711 \\
\hline jersey 0,29440 & vestido 0,37620 \\
\hline vestido 0,19971 & chaqueta 0,28609 \\
\hline vaquero 0,17031 & jersey 0,23966 \\
\hline corbata 0,16696 & vaquero 0,20621 \\
\hline bañador 0,16080 & calcetín 0,18753 \\
\hline chaqueta 0,15305 & gorra 0,18131 \\
\hline azul 0,13433 & sombrero 0,17779 \\
\hline negro 0,12322 & abrigo 0,12740 \\
\hline verde 0,10848 & corbata 0,12377 \\
\hline pantalón corto 0,09936 & cinturón 0,12105 \\
\hline blanco 0,09304 & gorro 0,10167 \\
\hline rojo 0,09110 & tienda 0,09291 \\
\hline calcetín 0,09013 & guante 0,08254 \\
\hline amarillo 0,08538 & bota 0,07798 \\
\hline bota 0,08099 & vestir 0,07688 \\
\hline
\end{tabular}

\begin{tabular}{|l|l|}
\hline \multicolumn{2}{|c|}{ 3. LA CASA } \\
\hline \multicolumn{1}{|c|}{ Estudiantes sec } & \multicolumn{1}{c|}{ Estudiantes uni } \\
\hline cocina 0,39910 & cocina 0,64480 \\
\hline cama 0,32826 & baño 0,35582 \\
\hline mesa 0,30521 & cama 0,35416 \\
\hline familia 0,28174 & mesa 0,32350 \\
\hline silla 0,21944 & ventana 0,32053 \\
\hline ventana 0,20010 & sofá 0,29168 \\
\hline sofá 0,19951 & puerta 0,28610 \\
\hline televisión 0,19653 & habitación 0,27548 \\
\hline puerta 0,18022 & dormitorio 0,26898 \\
\hline jardín 0,17314 & jardín 0,25561 \\
\hline
\end{tabular}




\begin{tabular}{|l|l|}
\hline ordenador 0,17088 & armario 0,25317 \\
\hline habitación 0,16140 & cuarto de baño 0,25082 \\
\hline baño 0,13697 & silla 0,22034 \\
\hline cuarto de baño 0,13283 & televisión 0,19420 \\
\hline grande 0,13163 & familia 0,17659 \\
\hline dormitorio 0,13020 & sillón 0,16248 \\
\hline padre 0,12048 & alfombra 0,15851 \\
\hline perro 0,11801 & techo 0,13712 \\
\hline armario 0,11439 & salón 0,12851 \\
\hline frigorífico 0,10806 & escalera 0,12221 \\
\hline
\end{tabular}

\begin{tabular}{|l|l|}
\hline \multicolumn{2}{|c|}{ 4. ALIMENTOS Y BEBIDAS } \\
\hline Estudiantes sec & \multicolumn{1}{|c|}{ Estudiantes uni } \\
\hline agua 0,49194 & agua 0,44756 \\
\hline cerveza 0,41266 & zumo 0,39249 \\
\hline paella 0,35042 & vino 0,33749 \\
\hline vino 0,29285 & cerveza 0,29987 \\
\hline zumo 0,26312 & leche 0,29672 \\
\hline fruta 0,24428 & pan 0,29478 \\
\hline verdura 0,23724 & carne 0,29087 \\
\hline tortilla 0,23645 & manzana 0,26167 \\
\hline carne 0,23504 & patata 0,25625 \\
\hline pan 0,22028 & fruta 0,24628 \\
\hline manzana 0,21848 & verdura 0,24303 \\
\hline pollo 0,20737 & plátano 0,23144 \\
\hline comer 0,19008 & naranja 0,21552 \\
\hline patata 0,18260 & tomate 0,21504 \\
\hline chorizo 0,17690 & café 0,19527 \\
\hline tomate 0,17504 & té 0,18771 \\
\hline café 0,17336 & paella 0,18046 \\
\hline naranja 0,16763 & fresa 0,15494 \\
\hline gazpacho 0,16681 & pescado 0,14757 \\
\hline leche 0,16590 & alcohol 0,14350 \\
\hline
\end{tabular}




\begin{tabular}{|c|c|}
\hline Estudiantes sec & Estudiantes uni \\
\hline calle 0,47485 & coche 0,54314 \\
\hline coche 0,45772 & gente 0,43822 \\
\hline casa 0,36803 & calle 0,43074 \\
\hline gente 0,30528 & casa 0,38100 \\
\hline tienda 0,30211 & (auto)bús 0,33879 \\
\hline parque 0,28552 & tráfico 0,31985 \\
\hline escuela 0,25156 & tienda 0,30873 \\
\hline (auto)bús 0,23856 & escuela 0,23722 \\
\hline plaza 0,21303 & ruido 0,23616 \\
\hline tráfico 0,20221 & parque 0,23320 \\
\hline cruzar 0,16191 & semáforo 0,17068 \\
\hline grande 0,12885 & edificio 0,16890 \\
\hline restaurante 0,10927 & bar 0,15039 \\
\hline monumento 0,10474 & restaurante 0,14941 \\
\hline árbol 0,10428 & carretera 0,14470 \\
\hline iglesia 0,10307 & tren 0,10749 \\
\hline mercado 0,09950 & atasco 0,10464 \\
\hline pasar 0,09704 & cine 0,10462 \\
\hline zona verde 0,09524 & ayuntamiento 0,09748 \\
\hline tren 0,08862 & bici(cleta) 0,09145 \\
\hline
\end{tabular}

\begin{tabular}{|l|l|}
\hline \multicolumn{2}{|c|}{ 6. EL CAMPO } \\
\hline \multicolumn{1}{|c|}{ Estudiantes sec } & \multicolumn{1}{c|}{ Estudiantes uni } \\
\hline vaca 0,52180 & animal 0,47635 \\
\hline animal 0,42613 & naturaleza 0,37834 \\
\hline naturaleza 0,32309 & árbol 0,35245 \\
\hline campesino 0,28639 & vaca 0,34916 \\
\hline caballo 0,27698 & campesino 0,32805 \\
\hline árbol 0,24523 & verde 0,29579 \\
\hline verde 0,22206 & tranquilidad 0,28222 \\
\hline bosque 0,18064 & bosque 0,26815 \\
\hline leche 0,17668 & casa 0,25714 \\
\hline
\end{tabular}




\begin{tabular}{|l|l|}
\hline perro 0,17380 & flor 0,21870 \\
\hline casa 0,17066 & paz 0,20714 \\
\hline sol 0,16268 & perro 0,17392 \\
\hline tranquilidad 0,12914 & sol 0,15965 \\
\hline tractor 0,12632 & río 0,14302 \\
\hline gato 0,12444 & caballo 0,13562 \\
\hline río 0,12127 & aire fresco 0,12938 \\
\hline campo 0,09821 & oveja 0,10823 \\
\hline abuelo 0,09679 & jardín 0,10791 \\
\hline flor 0,09367 & montaña 0,10720 \\
\hline verdura 0,09026 & gato 0,10501 \\
\hline
\end{tabular}

\begin{tabular}{|l|l|}
\hline \multicolumn{2}{|c|}{ 7. MEDIOS DE TRANSPORTE } \\
\hline \multicolumn{1}{|c|}{ Estudiantes sec } & \multicolumn{1}{c|}{ Estudiantes uni } \\
\hline coche 0,78178 & coche 0,84488 \\
\hline bici(cleta) 0,70439 & (auto)bús 0,78065 \\
\hline (auto)bús 0,64898 & tren 0,68147 \\
\hline avión 0,62315 & bici(cleta) 0,66521 \\
\hline tren 0,60550 & avión 0,62919 \\
\hline pie 0,34785 & barco 0,34260 \\
\hline barco 0,26180 & pie 0,24839 \\
\hline moto(cicleta) 0,18669 & moto(cicleta) 0,23721 \\
\hline taxi 0,12709 & taxi 0,21959 \\
\hline caballo 0,12375 & metro 0,18401 \\
\hline viajar 0,12220 & aeropuerto 0,09633 \\
\hline mar 0,10111 & viajar 0,08793 \\
\hline barca 0,08434 & tráfico 0,07575 \\
\hline tráfico 0,08350 & automóvil 0,07536 \\
\hline calle 0,08102 & carro 0,06962 \\
\hline conducir 0,07429 & calle 0,06921 \\
\hline metro 0,06977 & helicóptero 0,06605 \\
\hline atasco 0,06802 & billete 0,06248 \\
\hline automóvil 0,06732 & caballo 0,06133 \\
\hline dinero 0,06341 & tranvía 0,06064 \\
\hline
\end{tabular}




\begin{tabular}{|c|c|}
\hline \multicolumn{2}{|c|}{ 8. LOS ANIMALES } \\
\hline Estudiantes sec & Estudiantes uni \\
\hline perro 0,78613 & perro 0,80057 \\
\hline gato 0,77797 & gato 0,76880 \\
\hline vaca 0,52726 & vaca 0,57602 \\
\hline caballo 0,49408 & caballo 0,41627 \\
\hline pájaro 0,25988 & pájaro 0,40721 \\
\hline toro 0,23623 & pez 0,25031 \\
\hline araña 0,19546 & gallina 0,24891 \\
\hline oveja 0,17922 & elefante 0,22992 \\
\hline pez 0,14241 & oso 0,21006 \\
\hline avispa 0,13395 & oveja 0,20755 \\
\hline pollo 0,13295 & cerdo 0,19864 \\
\hline abeja 0,12963 & toro 0,19844 \\
\hline elefante 0,12909 & león 0,19224 \\
\hline mono 0,10431 & abeja 0,17757 \\
\hline serpiente 0,09966 & serpiente 0,17728 \\
\hline cerdo 0,09111 & conejo 0,15691 \\
\hline zoo(lógico) 0,08959 & ratón 0,15173 \\
\hline ratón 0,08052 & mariposa 0,14608 \\
\hline león 0,07386 & jirafa 0,14375 \\
\hline jirafa 0,07212 & tigre 0,13543 \\
\hline
\end{tabular}

\begin{tabular}{|l|l|}
\hline \multicolumn{2}{|c|}{ 9. JUEGOS Y DISTRACCIONES } \\
\hline \multicolumn{1}{|c|}{ Estudiantes sec } & \multicolumn{1}{c|}{ Estudiantes uni } \\
\hline fútbol 0,52916 & música 0,34433 \\
\hline baloncesto 0,51732 & tele(visión) 0,34230 \\
\hline música 0,30814 & fútbol 0,33982 \\
\hline jugar 0,29490 & libro 0,29107 \\
\hline nadar 0,25291 & baloncesto 0,27820 \\
\hline cine 0,21627 & amigo 0,26086 \\
\hline tele(visión) 0,21463 & deporte 0,22375 \\
\hline tenis 0,21268 & correr 0,20908 \\
\hline deporte 0,21223 & pasear 0,20861 \\
\hline
\end{tabular}




\begin{tabular}{|l|l|}
\hline voleibol 0,21179 & tenis 0,18343 \\
\hline amigo 0,21110 & cine 0,18142 \\
\hline correr 0,17973 & ajedrez 0,18096 \\
\hline ordenador 0,17116 & jugar 0,16280 \\
\hline bailar 0,12902 & leer 0,15533 \\
\hline balonmano 0,12593 & nadar 0,14936 \\
\hline bici(cleta) 0,11416 & bici(cleta) 0,14883 \\
\hline baile 0,10250 & ordenador 0,12964 \\
\hline fiesta 0,09937 & bailar 0,12900 \\
\hline discoteca 0,09220 & salir 0,12226 \\
\hline niño 0,08573 & cartas 0,11505 \\
\hline
\end{tabular}

\begin{tabular}{|l|l|}
\hline \multicolumn{2}{|c|}{ 10. PROFESIONES Y OFICIOS } \\
\hline Estudiantes sec & \multicolumn{1}{|c|}{ Estudiantes uni } \\
\hline profesor 0,65395 & profesor 0,82405 \\
\hline médico 0,35287 & médico 0,46630 \\
\hline actor 0,30911 & abogado 0,33593 \\
\hline doctor 0,26505 & traductor 0,29323 \\
\hline director 0,20662 & camarero 0,26317 \\
\hline cantante 0,19398 & policía 0,23885 \\
\hline actriz 0,17490 & secretario 0,22972 \\
\hline camarero 0,17462 & director 0,19355 \\
\hline policía 0,16611 & vendedor 0,18317 \\
\hline abogado 0,15928 & enfermero 0,17071 \\
\hline ama de casa 0,15799 & actor 0,16165 \\
\hline trabajar 0,15347 & albañil 0,16033 \\
\hline dinero 0,14217 & conductor 0,15175 \\
\hline secretario 0,14153 & cocinero 0,14415 \\
\hline arquitecto 0,12797 & ama de casa 0,13574 \\
\hline vendedor 0,12545 & peluquero 0,13203 \\
\hline cartero 0,11002 & juez 0,12084 \\
\hline oficina 0,10404 & deportista 0,12025 \\
\hline jefe 0,10336 & cantante 0,11382 \\
\hline bombero 0,10021 & bombero 0,11258 \\
\hline
\end{tabular}




\begin{tabular}{|l|l|}
\hline \multicolumn{2}{|c|}{ 11. ACCIONES QUE SE REALIZAN TODOS LOS DÍAS } \\
\hline \multicolumn{1}{|c|}{ Estudiantes sec } & \multicolumn{1}{c|}{ Estudiantes uni } \\
\hline comer 0,71652 & comer 0,72475 \\
\hline dormir 0,51250 & dormir 0,60436 \\
\hline estudiar 0,47903 & beber 0,47271 \\
\hline levantar 0,46471 & estudiar 0,45528 \\
\hline escuela 0,43689 & hablar 0,36838 \\
\hline beber 0,35331 & levantar 0,33071 \\
\hline tele(visión) 0,29254 & leer 0,27591 \\
\hline duchar 0,26821 & duchar 0,23567 \\
\hline hablar 0,25959 & lavar 0,21277 \\
\hline leer 0,15829 & tele(visión) 0,20740 \\
\hline amigo 0,15821 & acostar 0,19688 \\
\hline escribir 0,15037 & escribir 0,19260 \\
\hline música 0,15012 & caminar 0,19056 \\
\hline lavar 0,14622 & trabajar 0,18267 \\
\hline lavar los dientes 0,14548 & escuchar 0,17788 \\
\hline acostar 0,14492 & vestir 0,17489 \\
\hline trabajar 0,13491 & cocinar 0,16630 \\
\hline cocinar 0,13347 & despertar 0,16402 \\
\hline correr 0,12169 & lavar los dientes 0,15458 \\
\hline caminar 0,10541 & pensar 0,15383 \\
\hline
\end{tabular}

